\title{
Analysis of ciprofloxacin and gentamicin diffusion in Proteus mirabilis 018 biofilm by laser interferometry method
}

\author{
Michał Arabski ${ }^{\boxplus}$, Sławomir Wąsik², Magdalena Zych¹, Wioletta Łakomiec ${ }^{1}$ and Wiesław Kaca ${ }^{1}$ \\ 1Department of Microbiology, Jan Kochanowski University, Kielce, Poland; 2Department of Molecular Physics, Institute of Physics, Jan Kochanow- \\ ski University, Kielce, Poland
}

\begin{abstract}
Laser interferometry is a measurement technique used in physical sciences, with a potential for new applications in microbiology. Our previously studies, focused on the quantitative analysis of antibiotics diffusion through membranes or their releasing from gel structure, indicate that this method might be useful in analysis of substances diffusion across the bacterial biofilms. As antibiotic - biofilm interaction model, we tested above method for determination of ciprofloxacin or gentamicin diffusion through Proteus mirabilis 018 biofilm. Laser interferometry analysis of antibiotics diffusion showed that the amount of ciprofloxacin transported through mature biofilm is 1.9 times higher than gentamicin. It was correlated with lower level of gentamicin in compare to the level of ciprofloxacin in biofilm, which amounts were predicted in biofilm during diffusion process by laser interferometry method. We suggest that the analysis of antibiotic diffusion in biofilm might by helpful in evaluation of effectiveness of antibacterial agents.
\end{abstract}

Key words: laser interferometry, Proteus sp., biofilm, ciprofloxacin, gentamicin

Received: 31 October, 2013; revised: 25 November, 2013; accepted: 13 December, 2013; available on-line: 30 December, 2013

\section{INTRODUCTION}

Bacterial biofilms formed on the surfaces of tissues and biomaterials, play a key role in persistent infections. It is observed that the resistance of biofilms to antibiotics is increased 10-100 times compared with planktonic cells (Mah \& O’Toole, 2001). Actually, bacteria growing in a biofilm definitely develops increased resistance to antimicrobial agents. It has been suggested that drug resistance in biofilm might be associated with changing profiles of outer membrane proteins (OMP), increasing expression of multiple drug resistance pumps, induction $r p o S$-dependent process, or preventing the access of antibiotics to the bacterial cell by exopolysaccharide matrix or glycocalyx structure (Mah \& O’Toole, 2001). Biophysical properties of biofilm structure (exopolysaccharide matrix) associated with reduced susceptibility to antibiotics limits the effective eradication of bacteria forming biofilm (Stewart, 2003). It is suggested that only the surface layers of biofilm are exposed to a lethal dose of the antibiotic due to a reaction-diffusion barrier that prevents transport of the antibiotic into the biofilm. The quantitative evaluation of antibiotics diffusion within biofilm structure might play a crucial role in the analysis of predicted bacterial drug resistance. Bacteria, which do not have known genetic basis for resistance, might be protected against antibiotics in biofilm in contrast to planktonic cells, like a $\beta$-lactamase-negative strain of Klebsiella pneumoniae against ampicillin (Stewart \& Costerton, 2001; Andrel et al., 2000).

The biggest concern regarding drug penetration though biofilm structure is the lack of precise technique enabling quantitative and dynamics analysis of this process. Actually, studies of diffusion in biofilms have been limited to indirect measurements, such as CFU determination bioassay using antibiotic release from disc to microporous polycarbonate membrane covered with a biofilm (Andrel et al., 2000). Moreover, measurements based on substance indicators might change mass, structure or hydrophilic properties in comparision to native form of substances, for example, fluor-conjugated dextrans used in confocal laser microscopy (Lawrence et al., 1994). Taking into consideration above limitations, we present the application of the laser interferometry method as a novel technique for quantitative analysis of antibiotics diffusion/accumulation in biofilm. We tested above method for determination of ciprofloxacin or gentamicin diffusion through Proteus mirabilis O18 biofilm as antibiotic-biofilm interaction model.

\section{MATERIAL AND METHODS}

Bacteria growth, membrane and chemicals. Proteus mirabilis O18 (PrK 34/57) were obtained from the Czech National Collection of Type Cultures (Institute of Epidemiology and Microbiology, Prague). P. mirabilis O18 strain expressing constitutively DsRed 2 protein an ampicillin-sensitive was obtained by electroporation of $P$. mirabilis $\mathrm{O} 18$ with a $\mathrm{pDsRed} 2$ prokaryotic expression vector that encodes DsRed2 containing pUC plasmid replication origin (Clontech). The transformants were screened on Luria-Bertani (LB) agar with ampicillin (Stankowska et al., 2012). P. mirabilis strain was cultivated under aerobic conditions in $\mathrm{LB}$ medium $\left(37^{\circ} \mathrm{C}, \mathrm{pH} 7.4-7.6\right)$.

Polymeric nuclear track membrane (nucleopore) with pores diameter $0.9 \mu \mathrm{m}$ was purchased from Joint Institute for Nuclear Research in Dubna, Russia. Ciprofloxacin was purchased from Krka (Nove Mesto, Slovenia). Gentamicin was purchased from Sigma Aldrich (St. Louis, MO, USA).

Analysis of $P$. mirabilis biofilm formation by image J software. Biofilm of $P$. mirabilis was formed in LB medium for $24-96 \mathrm{~h}$ in stationary conditions at $37^{\circ} \mathrm{C}$ on

e-mail: arabski@ujk.edu.pl

*Part of the results was presented at the 3-rd Workshop on Microbiology "MIKROBIOT 2013" in Łódź, Poland.

Abbreviations: $\mathrm{CBL}$, concentration boundary layer; $\mathrm{CFU}$, colony forming unit; CV, crystal violet; LB, Luria-Bertani; LPS, lipopolysaccharide; OMP, outer membrane proteins; S.D., standard device. 
sterile nucleopore membrane with pores diameter 0.9 $\mu \mathrm{m}$ and area size $7 \times 10^{-5} \mathrm{~m}^{2}$, as a element of membrane system from laser interferometry equipment. After bacteria growth, nucleopore membranes with formed biofilm (after $24 \mathrm{~h}, 48 \mathrm{~h}, 72 \mathrm{~h}$ or $96 \mathrm{~h}$ ) were washed 3 times by $2 \mathrm{ml}$ of $0.9 \% \mathrm{NaCl}$ using manual pipette and were stained by crystal violet $(0.4 \%)$ for $15 \mathrm{~min}$. or analyzed by fluorescence microscopy. The microscopy images were collected (nine images of different areas from one membrane), converted to grey-scale digital images and saved in a tif file format. The images were imported to an Image J computer imaging software program (Schneider et al., 2012) to estimate the percentage degree of membrane covered by biofilm (i) and the mean of the greys from nine images for each membrane (ii). The value 1 denotes black colour and value 256 denotes white colour. Moreover, the membrane probes stained by $\mathrm{CV}$ were incubated with $30 \%$ acetate acid to release crystal violet from biofilm, and the level of the stain in supernatants was determined at $531 \mathrm{~nm}$ by Microplate Reader TECAN Infinite 200 PRO (Tecan Group Ltd., Switzerland) (Stefanovic et al., 2000). All tests were repeated 3 times.

Laser interferometry. The measurements of antibiotics diffusion through $P$. mirabilis $\mathrm{O} 18$ biofilm, as well as presence in biofilm structure, were measured by laser interferometry system. The system consists of a MachZehnder interferometer with a He-Ne laser, a membrane system (place of biofilm formation), a TV-CCD camera, and a computer with a system for the acquisition and processing of interference images (Arabski et al., 2007, 2009a, 2009b; Wasik et al., 2010). The laser light is spatially filtered and is transformed by the beam expander into a parallel beam about $80 \mathrm{~mm}$ wide and then split into two beams. The first beam goes through the investigated membrane with formed biofilm system, while the second goes directly through the compensation plate to the light detection system. As a consequence of the superimposition of these beams, respective interference images are generated. The images (interferograms) depend on the refraction coefficient of the solute, which in turn depends on the antibiotic concentration. When the solute is uniform the interference fringes are straight, and they bend when a concentration gradient appears. The concentration profile $C(x, t)$ is determined by the deviation of the fringes from a straight course. Since the concentration $C$ and the refraction coefficient are assumed to be linear, we have:

$$
C(x, t)=C_{0}+a \frac{\lambda d(x, t)}{h f},
$$

where $C_{0}$ is the initial antibiotic (ciprofloxacin or gentamicin) concentration $(1.5 \mathrm{mmol} / \mathrm{L}), a$ is the proportionality constant between the concentration and the refraction index $\left(a=6.60 \times 10^{3} \mathrm{~mol} / \mathrm{m}^{3}\right.$ and $9.47 \times 10^{3}$ $\mathrm{mol} / \mathrm{m}^{3}$ for the ciprofloxacin and gentamicin aqueous solution, respectively), $\lambda$ is the wavelength of the laser light, $b$ is the distance between the fringes in the field where they are straight lines, and $f$ is the thickness of the solution layer in the measurement cuvette. The CBL thickness was defined as the distance from the membrane with formed biofilm-water interface to the point where the deviation $d$ of the interference fringe from its straight line run is $10 \%$ of the fringe thickness. The interferograms are recorded from 120 to $2400 \mathrm{~s}$ with a time interval of $\Delta t=120 \mathrm{~s}$ and the profiles for substance are reconstructed. Such profiles were used to calculate the amount of antibiotic in function of time $(N(t))$ transported through biofilm P. mirabilis O18 formed for 96 h. Additionally, we calculated the amount of antibiotic into biofilm structure during diffusion of antibiotic for $40 \mathrm{~min}$. The amount of antibiotic inside the biofilm after time $t$ was calculated according to:

$$
N(t)=S \int_{0}^{l} C_{m}(x, t) d x,
$$

where $S$ and $l$ denote the surface $\left(7.0 \times 10^{-5} \mathrm{~m}^{2}\right)$, respectively, and thickness of membrane with biofilm, $C_{m}(x, t)$ is the concentration profile inside the membrane with formed biofilm. We assumed the linear concentration distribution (Hoogervorst et al., 1978):

$$
C_{m}(x, t)=\frac{C_{2}(l, t)-C_{1}(0, t)}{l} x,
$$

where $C_{1}(0, t), C_{2}(l, t)$ are the concentration values on both surfaces of biofilm membrane. $C_{1}(0, t), C_{2}(l, t)$ and $l$ were determined interferometrically.

Moreover, the thickness of biofilm was measured by laser interferometry method. The resolution of camera (element of laser interferometer system) determined the measuring accuracy: in our conditions 1 pixel $=0.019$ $\mathrm{mm}$.

Data analysis. The data were analysed using the Statistica version 10 (StatSoft, Tulsa, OK, USA) software package. The values of grey level determined by imageJ software and absorbance are expressed as the mean \pm S.D. from three independent experiments. P values less than 0.05 were considered statistically significant. The differences were compared by the ANOVA test and post-hoc (Tukey) test.

\section{RESULTS}

\section{Analysis of $P$. mirabilis biofilm formation}

Figure 1A shows the examples of nucleopore membranes images with formed biofilm in LB medium at $37^{\circ} \mathrm{C}$, stained by crystal violet and converted to greyscale. Analysis of nine microscopy images for each membrane with formed biofilm for 24-96 h by imageJ software (Fig. 1B), as well as spectrometrically determination of crystal violet total released from whole membranes (Fig. 1C), indicate that P. mirabilis $\mathrm{O} 18$ formed statistically significant biofilm after $72 \mathrm{~h} / 96 \mathrm{~h}$ (ANOVA and Tukey tests analyses). For laser interferometry analysis, we used biofilm formed for $96 \mathrm{~h}$ in $\mathrm{LB}$ medium at $37^{\circ} \mathrm{C}$. Moreover, image analysis of the percentage degree of membrane covered by biofilm shows that nucleopore membrane is covered in $78.55 \pm 8.17 \%$ by biofilm $P$. mirabilis $\mathrm{O} 18$ after $96 \mathrm{~h}$ in $\mathrm{LB}$ medium at $37^{\circ} \mathrm{C}$. Figure 2 shows the example of biofilm image analysis by image in different time intervals of incubation $(24,48,72$ and $96 \mathrm{~h}$ ). Figure 3 shows the examples of membrane images obtained by fluorescence microscopy. Analysis of expressing constitutively DsRed 2 protein in biofilm formed bacterial cells shows that membrane is covered in $72.35 \pm 6.22 \%$ by biofilm $P$. mirabilis $\mathrm{O} 18$ after $96 \mathrm{~h}$ in LB medium at $37^{\circ} \mathrm{C}$. The thickness of biofilm was ca. $0.057 \mathrm{~mm}$ on nucleopore membrane calculated by a laser interferometry method. 

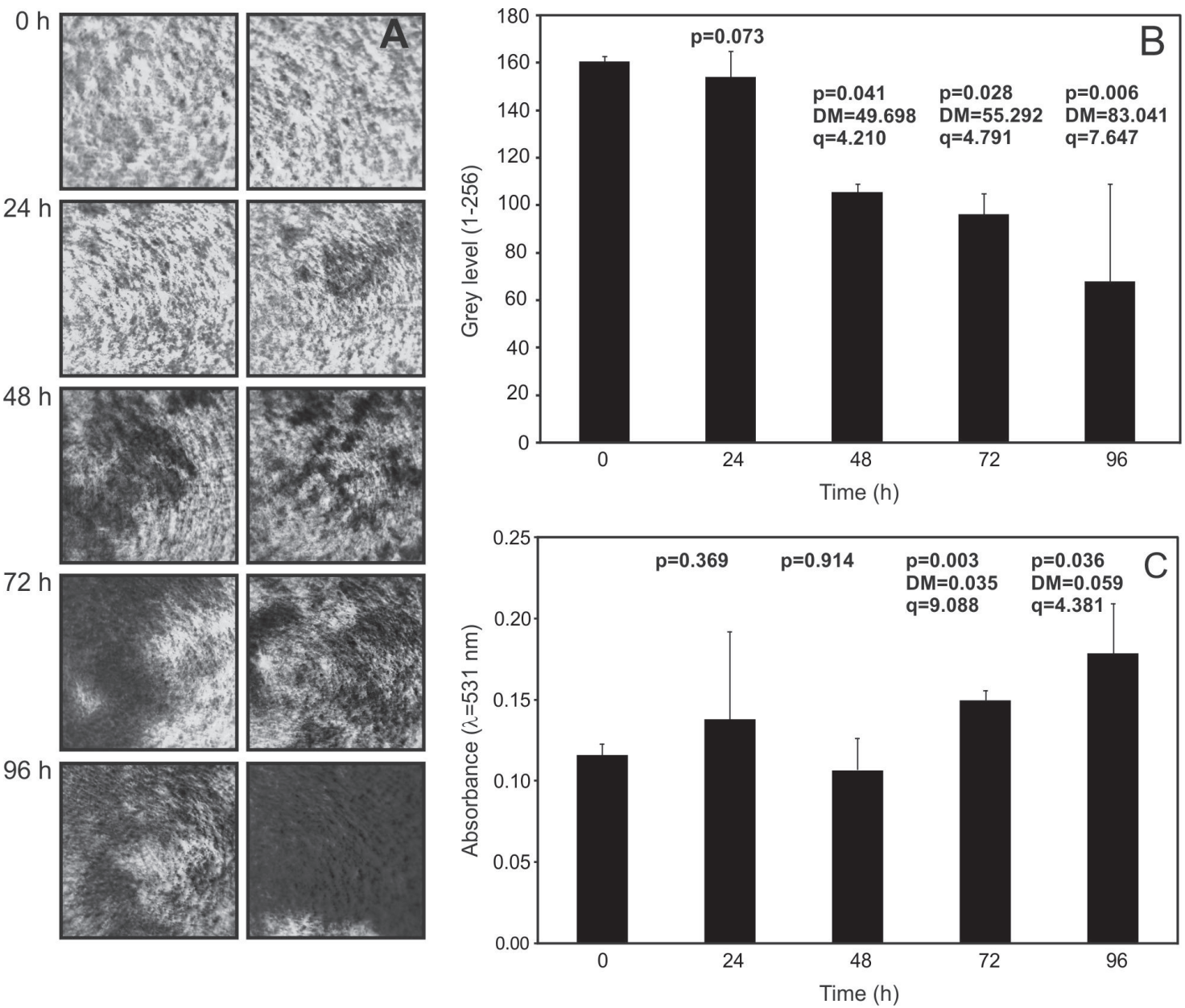

Figure 1. The examples of nucleopore membranes images with formed $P$. mirabilis 018 biofilm in LB medium for 24-96 $\mathrm{h}$ in stationary conditions at $37^{\circ} \mathrm{C}$ stained by crystal violet (Panel A). Panel B shows the means of grey levels of nine images of different areas for each membrane (time of biofilm growth) determined by imageJ software. Panel C shows the levels of released crystal violet from membranes with biofilm formed for 24-96 h determined spectrometrically at $531 \mathrm{~nm}$.

The results of ANOVA and Tukey tests analyses are marked (DM - difference in means).

\section{Analysis of ciprofloxacin distribution in biofilm using laser interferometry}

Figure 4 shows the amount of antibiotics transported through biofilm $P$. mirabilis O18 formed on nucleopore membrane for $96 \mathrm{~h}$ at the initial concentrations $1.5 \mathrm{mmol} / \mathrm{L}$. We observed that the amount of transported ciprofloxacin and gentamicin were $4.55 \times 10^{-8}$ mol and $2.44 \times 10^{-8}$ mol after $40 \mathrm{~min}$, respectively. Additionally, laser interferometry method was used to

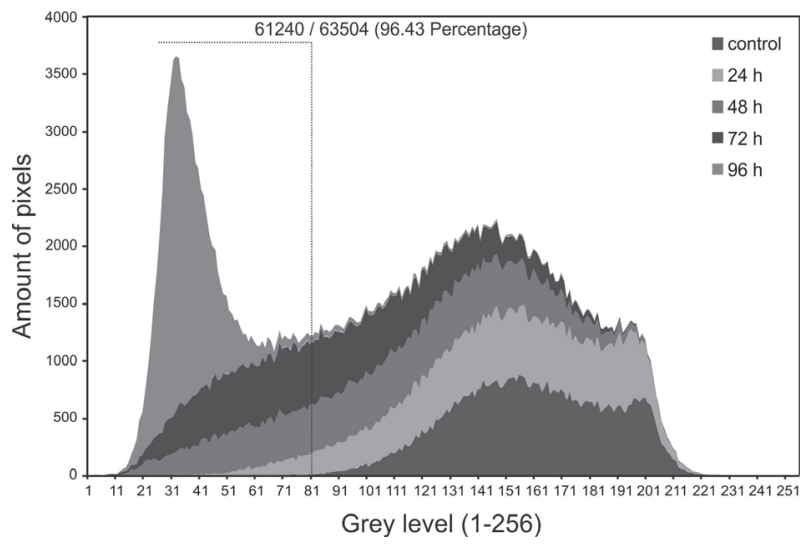

calculate the amount of antibiotics present in the biofilm during diffusion. Figure 4 shows that the amount of ciprofloxacin increased from $1.28 \times 10^{-8} \mathrm{~mol}$ to $1.86 \times 10^{-8} \mathrm{~mol}$ at $2 \mathrm{~min}$ and $40 \mathrm{~min}$, respectively after antibiotic exposition. It indicated that the amount of ciprofloxacin maintained in biofilm was about $1.57 \times 10^{-8} \mathrm{~mol}$ during diffusion process (40 min). For gentamicin, we observed that the amount of antibiotic in biofilm during diffusion is lower and rather constant (ca. $5.82 \times 10^{-10} \mathrm{~mol}$ ). The kinetics of two antibiotics diffusion through $P$. mirabilis O18 biofilm differs significantly. Ciprofloxacin diffuses in constant manner contrary to gentamicin, which after $10 \mathrm{~min}$. reaches constant value. It was about $50 \%$ less than ciprofloxacin after $40 \mathrm{~min}$.

Figure 2. Histograms of imageJ software analysis (percentage degree of membrane covered by biofilm) of representative biofilm images for each membrane formed in LB medium for 24-96 $\mathrm{h}$ in stationary conditions at $37^{\circ} \mathrm{C}$ and stained by crystal violet.

Grey line presents cut-off point in which 61240 pixels from total analyzed 63540 have the grey level lower than 81 . The value 81 is the most grey level determined for control membrane by imageJ. Percentage of membrane covering by biofilm formed for $96 \mathrm{~h}$ is presented. 

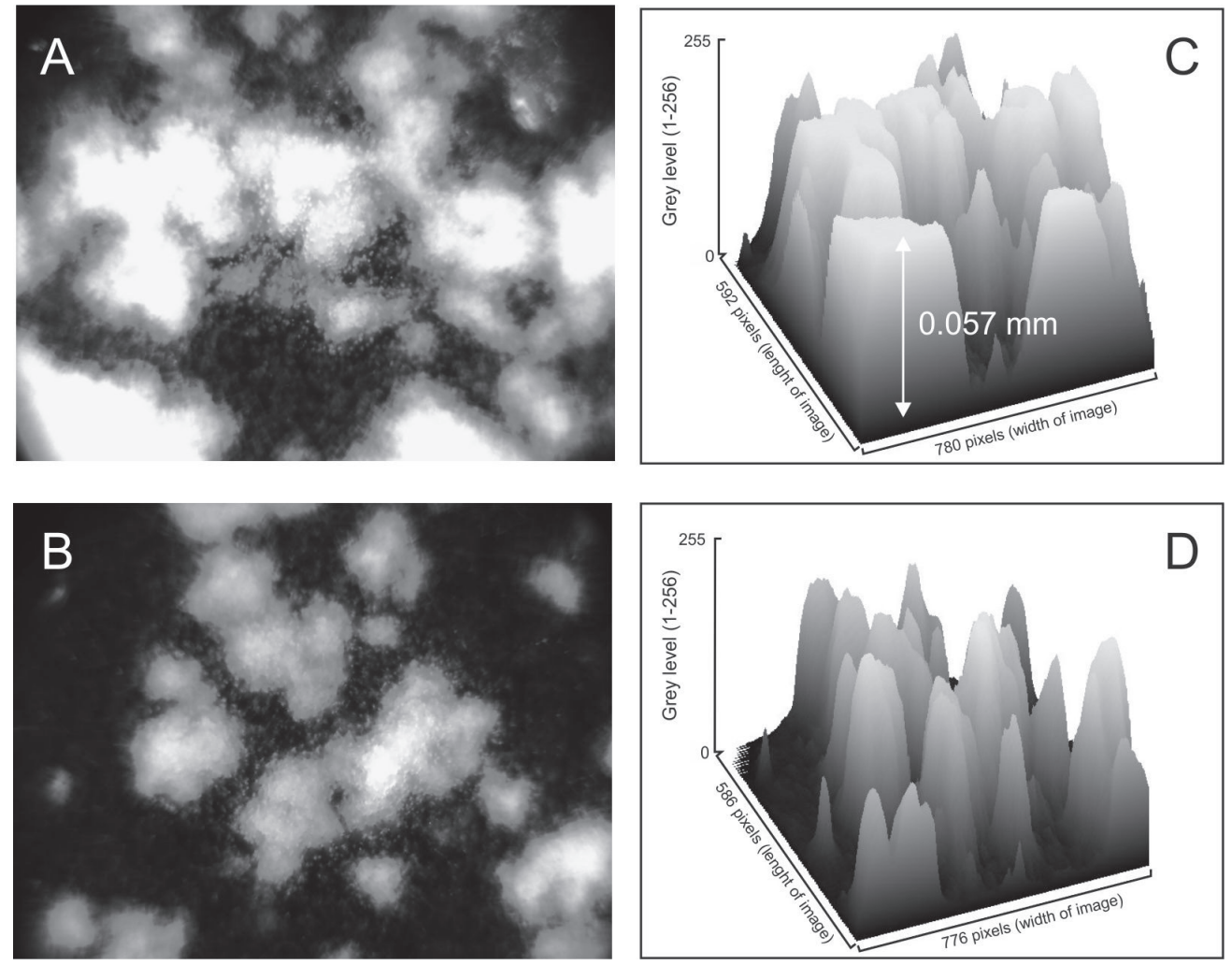

Figure 3. The examples (Panels A and B) of nucleopore membranes images with formed P. mirabilis 018 biofilm in LB medium for 96 $\mathrm{h}$ in stationary conditions at $37^{\circ} \mathrm{C}$ obtained by fluorescence microscopy. Panels $\mathrm{C}$ and $\mathrm{D}$ shows the surface plots of images $\mathrm{A}$ and $\mathrm{B}$, respectively, by imageJ software.

The biofilm thickness measured by laser interferometry method is indicated.

\section{DISCUSSION}

Laser interferometry is a measurement technique used in physical sciences, with a potential for new applications in microbiology. This method, based on the phenomenon of wave interference, enables quantitative substance assays by means of measurement of the difference be-

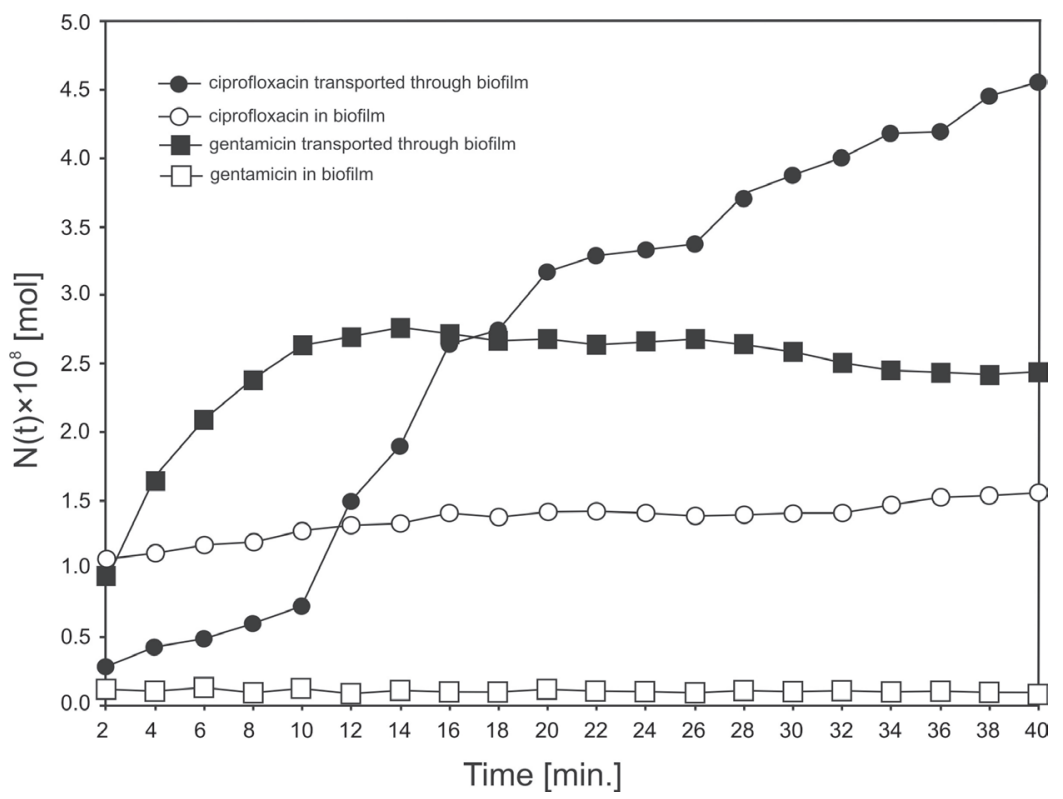

Figure 4. The amount of ciprofloxacin or gentamicin transported through P. mirabilis 018 biofilm or predicted in its structure by laser interferometry method. Biofilm was formed for $96 \mathrm{~h}$ in LB medium in stationary conditions at $37^{\circ} \mathrm{C}$. tween light refractive indexes for the studied and control substances. This technique was previously used for physical research of diffusion process, like characteristics of membrane permeability and analysis of concentration layer evolution in near-membrane fields, anomalous substance diffusion in gel structure, or assessment of hydrodynamic instabilities on the effectiveness of a diffusion process (Dworecki et al., 1995, 1997, 2003, 2005, 2006). At present, the literature data pertaining to applications of laser interferometry in biological sciences is scarce. Until now, this kind of analysis was used in microbiology for the analysis of metabolic activity of Mycobacteria (Machado et al., 2008), lipopolysaccharide (LPS) interaction with biologically active compounds (Arabski et al., 2007, 2009a, 2009b), analysis of antibiotic and liposomes diffusion across membranes (Arabski et al., 2012b) or hydrophobicity assessment of LPS isolates in order to optimise the ELISA method (Arabski et al., 2012a). Generally, above applications of laser interferometry technique in quantitative analysis of antibiotics diffusion through membranes, or their releasing from gel structure indicate that this method might be useful in analysis of substances diffusion across the bacterial biofilms.

The first step of our investigation was optimization of $P$. mirabilis $\mathrm{O} 18$ tight biofilm formation on nucleopore 
membrane with pore diameter $0.9 \mu \mathrm{m}$ as a "scaffolding" for formed biofilm. On the basis of imageJ software analyses of CV staining biofilm (Figs. 1, 2) and fluorescence of DsRed2 protein expressing in biofilm formed bacterial cells (Fig. 3), we observed that P. mirabilis O18 formed statistically significant $(p<0.05)$ tight biofilm (thickness $0.057 \mathrm{~mm}$ ) after $96 \mathrm{~h}$ and covered membrane in about $76 \%$. Laser interferometry analysis of antibiotics diffusion (Fig. 4) showed that the amount of ciprofloxacin transported through mature biofilm is 1.9 times higher than gentamicin $\left(4.55 \times 10^{-8} \mathrm{~mol} / 2.44 \times 10^{-8} \mathrm{~mol}\right)$. It was correlated with lower level of gentamicin in compare to the level of ciprofloxacin in biofilm, which amounts were predicted in biofilm during diffusion process by the laser interferometry method. It indicated that the worse penetration of gentamicin in contrast to ciprofloxacin in biofilm might be positively correlated with antibiotic susceptibility patterns observed for clinical strains: 90\% bacterial strains were resistant to gentamicin and 30\% for ciprofloxacin (Christopher et al., 2013) or in another study, $70 \%$ strains isolated from urinary tract infection were resistant to gentamicin and $50.5 \%$ for ciprofloxacin (Otajevwo, 2013).

In conclusion, laser interferometry method might be a useful technique in analysis of the biophysical properties of bacterial biofilms, important from the clinical point of view. The characteristic of antibiotic diffusion in biofilm might by helpful in evaluation of effectiveness of the antibacterial agents.

\section{Acknowledgments}

We would like to thank Natalia Góźdź for a technical support and Dorota Stańkowska for making the P. mirabilis $\mathrm{O} 18$ with cloned pDsRed2 available in this study.

This work was supported by grant NN 304275540 from NRC, Poland and BS 2013 from Jan Kochanowski University.

\section{REFERENCES}

Anderl JN, Franklin MJ, Stewart PS (2000) Role of antibiotic penetration limitation in Klebsiella pneumoniae biofilm resistance to ampicillin and ciprofloxacin. Antimicrob Agents Chemother 44: 1818-1824.

Arabski M, Fudała R, Wąsik S, Koza A, Futoma-Kołoch B, BuglaPłoskońska G, Kaca W (2012a) The presence of anti-LPS antibodies, human serum activity against $P$. mirabilis $\mathrm{S} / \mathrm{R}$ forms and TLR4 (Thr399Ile) gene polymorphism in rheumatoid arthritis. Clin Biochem 5: $1374-1382$.

Arabski M, Davydova VN, Wąsik S, Reunov AV, Lapshina LA, Solov eva TF, Yermak IM, Kaca W (2009a). Binding of lipopolysaccharide from Proteus vulgaris O25 (48/57) with chitosan. Carbobydr Polym 78: 481-487.
Arabski M, Wąsik S, Dworecki K, Kaca W (2007) Laser interferometric determination of ampicillin and colistin transfer through cellulose biomembrane in the presence of Proteus vulgaris O25 lipopolysaccharide. J Membrane Sci 299: 268-275.

Arabski M, Wąsik S, Dworecki K, Kaca W (2009b) Laser interferometric and cultivation methods for measurement of colistin/ampicilin and saponin interactions with smooth and rough of Proteus mirabilis lipopolysaccharides and cells. J Microbiol Methods 77: 179-183.

Arabski M, Wassik S, Grześkiewicz H, Drulis-Kawa Z, Gubernator J, Kaca W (2012b) Laser interferometric determination of liposome diffusion through artificial membranes, Chapter 14 in the monograph entitled Interferometry - Research and Applications in Science and Technology, ISBN: 978-953-51-0403-2.

Christopher A, Mshana SE, Kidenya BR, Hokororo A, Morona D (2013) Bacteremia and resistant gram-negative pathogens among under-fives in Tanzania. Ital J Pediatr 8: 27.

Cleveland JP, Manne S, Bocek D, Hansma PK (1993) A non-destructive method for determining the spring constant of cantilevers for scanning force microscopy. Rev Sci Instrum 64: 403-405.

Dworecki K (1995) Interferometric investigation of near-membrane diffusion layers. J Biol Phys 21: 37-49.

Dworecki K (2006) Experimental investigation of the subdiffusion in a membrane system. Physica A 359: 24-32.

Dworecki K, Ślęzak A, Ornal-Wąsik B., Wąsik S (2005) Effect of hydrodynamic instabilities on solute transport in a membrane system. J Memb Sci 265: 94-100.

Dworecki K, Wasik S (1997) The investigation of time-dependent solute transport through horizontally situated membrane: the effect of configuration membrane system. J Biol Phys 23: 181-195.

Dworecki K, Wąsik S, Ślęzak A (2003) Temporal and spatial structure of the concentration boundary layers in a membrane system. Physica A 326: 360-369.

Hoogervorst CJP, De Goede J, Versluis CW, Smith JAM (1978) Nonstationary diffusion through membranes. 2. Transient diffusion through a membrane separating two semiinfinite volumes of unstirred solutions. J Phys Chem 82: 1318-1324.

Lawrence JR, Wolfaardt GM, Kober DR (1994) Determination of diffusion in biofilms by confocal laser microscopy. Appl Environ Microbiol 60: 1160-1173.

Machado RR, Lima Filho ES, Jardim DF, Ferreira MA, de Faria CG, Duarte RS, Lesche B (2008) Metabolic activity interferometer: description and calibration of an interferometric method to measure growth of mycobacteria. Eur Biophys J 38: 111-119.

Mah TFC, O’Toole GA (2001) Mechanisms of biofilm resistance to antimicrobial agents. Trends Microbiol 9: 34-39.

Otajevwo FD.(2013) Urinary tract infection among symptomatic outpatients visiting a tertiary hospital based in midwestern Nigeria. Glob J Health Sci 13:187-99.

Schneider CA, Rasband WS, Eliceiri KW (2012) NIH Image to ImageJ: 25 years of image analysis. Nat Methods 9: 671-675.

Stańkowska D, Czerwonka G, Różalska S, Grosicka M, Dziadek J, Kaca W (2012) Influence of quorum sensing signal molecules on biofilm formation in Proteus mirabilis O18. Folia Microbiol 57: 53-60.

Stepanovic S, Vukovic D, Dakic I, Savic B, Svabic-Vlahovic M (2000) A modified microtiter-plate test for quantification of staphylococcal biofilm formation. J Microbiol Methods 40: 175-179.

Stewart PS (2003) Diffusion in biofilms. J Bacteriol 185: 1485-1491.

Stewart PS, Costerton JW (2001) Antibiotic resistance of bacteria in biofilms. Lancet 356: 135-138.

Wąsik S, Arabski M, Dworecki K, Ślęzak A, Kaca W (2010) Influence of gravitational field on the substance transport in gels. I Membrane Sci 365: 341-346. 